\title{
Buckling mode interaction in prestressed stayed columns
}

\author{
M. Ahmer Wadee, Leroy Gardner and Tyrone A. Hunt \\ Department of Civil \& Environmental Engineering, \\ Imperial College London, London SW7 2AZ, UK
}

\begin{abstract}
Prestressed stayed columns offer an innovative and aesthetically attractive solution where designs demand long and slender elements under compression. The addition of cross-arms and pretensioned cables to the columns provide significant lateral and rotational support that can result in a marked increase of the buckling capacity. The current work presents a simplified model, comprising discrete rigid-links and springs with a mathematically exact formulation including the destabilizing effect of cable slackening, which mimics the response of stayed columns and reveals the governing physical parameters that drive the behaviour. A recent finite element study had indicated that these structural components are likely to exhibit interactive buckling phenomena, particularly if the critical buckling mode is antisymmetric, leading to highly unstable responses. However, by its very nature, that study could not reveal the bifurcational structure of the equilibrium response. The results from the present model reveal similar behaviour to that observed in the earlier finite element study. A sequence of bifurcations is found that switch the post-buckling response from one mode to another via a path of interactive buckling.
\end{abstract}

\section{Notation}

$\begin{array}{ll}a & \text { Length of one cross-arm } \\ A, A_{s} & \text { Cross-sectional area of main column and stay respectively } \\ c, c_{0} & \text { Stiffness of rotational springs of main column } \\ E, E_{a}, E_{s} & \text { Young's modulus of main column, cross-arm and stay respectively } \\ I, I_{a} & \text { Second moment of area of main column and cross-arm respectively } \\ k, K & \text { Actual and non-dimensional stiffness of longitudinal spring: } K=k l^{2} / c \\ l & \text { Length of an individual link in the model } \\ L & \text { Length of main column } \\ L_{s}, L_{s i} & \text { Length of an individual stay or stay } i \\ L_{v i} & \text { Vertical length component of stay } i \\ m_{i} & \text { Shape scaling factor of an eigenmode }\end{array}$




$\begin{array}{ll}P, p & \text { Actual and non-dimensional axial load: } p=P l / c \\ P_{i}^{\mathrm{C}}, p_{i}^{\mathrm{C}} & \text { Actual and non-dimensional critical buckling loads of mode } i: p_{i}^{\mathrm{C}}=P_{i}^{\mathrm{C}} l / c \\ Q_{i}, u_{i} & \text { Generalized coordinates of link model } \\ R & \text { Ratio of rotational spring stiffnesses: } c_{0} / c \\ S & \text { Shape of an eigenmode } \\ \mathrm{S}_{i} & \text { Labels of bifurcation points } \\ T & \text { Prestressing force in stays } \\ X & \text { Non-dimensional quantity: } \sqrt{8-4 K+K^{2}} \\ V, \tilde{V} & \text { Actual and non-dimensional total potential energy: } \tilde{V}=V / c \\ \mathcal{E}, \tilde{\mathcal{E}} & \text { Actual and non-dimensional end-displacement in link model: } \tilde{\mathcal{E}}=\mathcal{E} / l \\ \alpha & \text { Initial angle of stays to the vertical } \\ \lambda_{i} & \text { Diagonalization factors } \\ \theta_{B}, \theta_{C}, \theta_{D} & \text { Rotation angles in the link model } \\ \theta_{c} & \text { Rotation angle of the cross-arm to the horizontal } \\ \phi_{s} & \text { Cross-section diameter of stay }\end{array}$

\section{Keywords}

Columns; Cables and tendons; Mathematical modelling; Engineering mechanics

\section{Introduction}

Prestressed stayed columns are increasingly being used as a solution to long-span structures where slender geometry and high strength are key design variables. A schematic and an example of such a column is shown in Figure 1. This type of component comprises a main column element, which is usually of tubular cross-section, a series of cross-arms and pre-tensioned cable stays that provide significant extra compressive strength without a commensurate increase in self-weight (Belenya, 1977; Smith et al., 1975; Temple, 1977; Hafez et al., 1979; Howson \& Williams, 1980; Wong \& Temple, 1982; Chan et al., 2002; De Araujo et al., 2008; Saito \& Wadee, 2008; Saito \& Wadee, 2010). These components are increasingly being used in the construction sector because of that advantage and because they have an aesthetic quality that is architecturally attractive, see Figure 1(b). They have also been used for shoring applications in the construction of the "Rock in Rio" stadium (Andrade et al., 2003), where an inexpensive, long and strong column was desired over an expensive trestle supporting structure. They have also been used to support the roof structure of the Frick Chemistry Building in Princeton University, USA (Seward, 2011).

However, precisely because these components are structurally efficient they have also been shown to be sensitive to initial imperfections in their geometry (Saito \& Wadee, 2009a). In particular, this sensitivity has been demonstrated in a recent finite element study (Saito \& Wadee, $2009 \mathrm{~b}$ ), which used different initial imperfection profiles of equivalent magnitudes to determine the weakest equilibrium response. However, by its very nature, that study could not reveal the bifurcational structure of the equilibrium response. The aim of the current work is to present the 
results from a simplified model that has a mathematically exact formulation, comprising rigid links and springs, which mimics the response and reveals the governing physical parameters that drive the behaviour. It is found, as in Saito \& Wadee (2009b) and Osofero et al (2012; 2013), that interactive buckling, which is triggered at a secondary bifurcation, does not occur until the antisymmetric mode becomes the critical eigenmode. This occurs when the strut is restrained at midspan by a translational spring of sufficient stiffness.

A mode interaction loop is found where the behaviour passes from the fundamental state to the initial critical buckling state, which then encounters the secondary bifurcation that leads to a path of interactive buckling followed by a subsequent bifurcation that returns to the path of a different eigenmode. The incorporation of stay slackening into the analytical model, by introducing a geometric constraint, distorts the interaction loop. Moreover, it is shown that with stay slackening, for higher lateral stiffnesses, the interaction loop breaks and that the strut would remain in the interactive mode after the secondary instability; exactly as found in the previous finite element study.

The paper begins with a review of the key previous work presented by Saito \& Wadee (2009b). The analytical model is then presented, followed by the numerical results that were determined by solving the developed equilibrium equations numerically by the powerful continuation package AUTO (Doedel \& Oldeman, 2009). The results are then compared with the original results from the earlier finite element study and are discussed. Conclusions, along with suggestions for further work, are then made.

\section{Review of finite element study}

The prestressed stayed column has distinctive features. In its most common form, it has a main column element of length $L$, a single cross-arm of length $a$ and cable stays that link the top and the bottom of the main column to the ends of the cross-arm, as shown in Figure 1. The cable stays, when pre-tensioned, provide the resisting horizontal components of force that restrain the potential buckling displacement at midspan. Since the cross-arm is rigidly connected to the column, the local bending stiffness is increased somewhat. The key work presented by Saito \& Wadee (2009b) contained a nonlinear finite element study on imperfect prestressed columns, formulated with Timoshenko beam elements for the main column and truss elements with the "no compression" option for the stays, which used different shapes of imperfections to trigger different post-buckling modes for stayed columns with varying geometries. The cross-arm length $a$ was varied, as was the diameter of the cable stays $\phi_{s}$; increasing either increased the critical load of the simplest eigenmode (Mode 1) that initially resembled a half-sine wave along the length. At a transition value for either $a$ or $\phi_{s}$, the first two eigenmodes coincided and for higher values, the second eigenmode (Mode 2) became critical. Note that the buckling eigenmodes are in-plane although given an out-of plane arrangement, the critical loads have been shown to be directionally invariant in earlier work if circular or rectangular hollow sections are used for the main column element (Saito \& Wadee, 2009a). Figure 2 shows graphs of the critical loads of the first two eigenmodes from the previous study showing where the transitions occur and Figure 3(b) defines the shapes of Modes 1 and 2. 
In the nonlinear analysis of the imperfect stayed columns, the primary observation was that interactive buckling was only a significant problem when Mode 2 was critical; when Mode 1 was critical, the worst case imperfection remained affine to the Mode 1 eigenmode. In the present study, the aim is to use a simplified analytical model to determine why this happens.

\section{Analytical model development}

The features of restraints and identifiable increases in stiffness from the stays and the cross-arms in the general arrangement lend themselves to a model comprising rigid links and linear elastic springs for the strut, as shown in Figure 3(a). The advantage of this being that the total potential energy for the system $V$ can be written in an exact form that allows numerical solutions of the equilibrium equations. These solutions can be obtained by AUTO (Doedel \& Oldeman, 2009), a powerful numerical continuation package which can compute locations of bifurcation points and separate solution branches. A three degree-of-freedom model is formulated comprising 4 rigid links each of length $l$, rotational springs at pins $B$ and $D$ of stiffness $c$ with a further rotational spring at pin $C$ of stiffness $c_{0}$ alongside a translational spring of stiffness $k$.

The stiffnesses $c, c_{0}$ and $k$ correspond to the bending of the main column, the inclusion of the cross-arm and the mechanical resistance of the pre-tensioned cable stays respectively. Expressions for the rotational springs may be calculated with the standard stiffness method of structural analysis (Coates et al., 1987), where the contributions from the main column element and the cross-arm are incorporated. These would comprise expressions of the form $x E I / L$ and $y E_{a} I_{a} / a$ derived from the contributions of the main column and the cross-arm respectively, where $E I$ and $E_{a} I_{a}$ are the respective flexural rigidities with $x$ and $y$ are evaluated using the aforementioned stiffness method. For the longitudinal spring of stiffness $k$ the summation of the horizontal components of the axial stiffnesses of the stays would give the total stiffness. The individual contribution of a stay is given by the expression $\left(E_{s} A_{s} / L_{s}\right) \sin \alpha$, where $E_{s}$ and $A_{s}$ are the Young's modulus and cross-sectional area of the stay respectively with $\alpha$ shown in Figure 1(a). It turns out that the stiffness $k$ has a more significant effect than the rotational springs on the overall behaviour of the model.

The external axial load is $P$ and the total potential energy is hence given by:

$$
V=\frac{1}{2} k l^{2} Q_{2}^{2}+\frac{1}{2} c\left(\theta_{B}^{2}+\theta_{D}^{2}\right)+\frac{1}{2} c_{0} \theta_{C}^{2}-P \mathcal{E},
$$

where $\mathcal{E}$ is the end-shortening and the angles $\theta_{B}, \theta_{C}$ and $\theta_{D}$ are the rotations of the pins at $B, C$ and $D$ respectively:

$$
\begin{aligned}
\mathcal{E} & =l\left[4-\sqrt{1-Q_{1}^{2}}-\sqrt{1-\left(Q_{2}-Q_{1}\right)^{2}}-\sqrt{1-\left(Q_{2}-Q_{3}\right)^{2}}-\sqrt{1-Q_{3}^{2}}\right] \\
\theta_{B} & =\arcsin \left(Q_{1}\right)+\arcsin \left(Q_{2}-Q_{1}\right), \\
\theta_{C} & =\arcsin \left(Q_{2}-Q_{1}\right)-\arcsin \left(Q_{2}-Q_{3}\right), \\
\theta_{D} & =\arcsin \left(Q_{3}\right)-\arcsin \left(Q_{2}-Q_{3}\right) .
\end{aligned}
$$


The total potential energy can be nondimensionalized by dividing through by $c$ :

$$
\tilde{V}=\frac{1}{2} K Q_{2}^{2}+\frac{1}{2}\left(\theta_{B}^{2}+\theta_{D}^{2}\right)+\frac{1}{2} R \theta_{C}^{2}-p \tilde{\mathcal{E}}
$$

with normalized quantities: $\tilde{V}=V / c, K=k l^{2} / c, R=c_{0} / c, p=P l / c$ and $\tilde{\mathcal{E}}=\mathcal{E} / l$. It can also be diagonalized and the resulting linear eigenvalue problem (see Appendix A for details) can be solved to obtain the following non-dimensional critical loads $p_{i}^{\mathrm{C}}$ for the case where $i=1,2,3$ and $R=1$ (assumed throughout the current work, i.e. the case when all the rotational springs are of identical stiffnesses):

$$
\begin{aligned}
& p_{1}^{\mathrm{C}}=\frac{K^{3}-K^{2}(X-2)+2 K(X-8)+16(3-X)}{2 X(X-2)}, \\
& p_{2}^{\mathrm{C}}=2, \\
& p_{3}^{\mathrm{C}}=\frac{K^{3}+K^{2}(X+2)-2 K(X+8)+16(3+X)}{2 X(X+2)},
\end{aligned}
$$

where the non-dimensional quantity $X=\sqrt{8-4 K+K^{2}}$. The linear eigenmodes have either reflective or rotational symmetry, as shown in Figure 3(b) and are given in Figure 4. The variation of the critical loads with the non-dimensional stiffness of the translational spring $K$ is shown in Figure 5. Note the similarity between the distribution of $P_{1}^{\mathrm{C}}$ and $P_{2}^{\mathrm{C}}$ in Figure 5 and the corresponding critical loads in the previous study (Saito \& Wadee, 2009b) as shown in Figure 2. This gives confidence in the model and allow the study to proceed. Of course more degrees of freedom could be used in the model; however, since it was shown in earlier work (Saito \& Wadee, 2008) that the symmetric and antisymmetric modes can be accurately represented by the first three modes, the current model has been restricted to three degrees of freedom. This reduces the complexity for the numerical solver while modelling the key features of mode interaction.

\subsection{Inclusion of stay slackening}

In a prestressed stayed column the stays are in tension when the component is deployed. Under axial compression, this prestress is relieved somewhat but in most cases not totally. However, if buckling occurs, the resulting lateral displacements prior to the instability can cause a rapid reduction of the cable tension on the concave sides of the buckling deformation due to the change in the geometric profile. Of course if the stay tension is relieved altogether, its stiffness drops effectively to zero and the load-carrying capacity of the entire component would reduce substantially. It is therefore necessary to include this geometric effect of potential stay slackening within the current model. With the numbering of the stays being defined in Figure 6(a), Figure 6(b) shows the geometry of the link model and how that relates to the stay lengths for arbitrary values of the generalized coordinates $Q_{i}$.

Geometrically, the vertical component of the lengths of the top two stays ( 1 and 4$)$ is:

$$
L_{v 1}=l\left[\sqrt{1-\left(Q_{2}-Q_{1}\right)^{2}}+\sqrt{1-Q_{1}^{2}}\right]
$$


and the bottom two stays (2 and 3$)$ is:

$$
L_{v 2}=l\left[\sqrt{1-\left(Q_{2}-Q_{3}\right)^{2}}+\sqrt{1-Q_{3}^{2}}\right] .
$$

Incorporating the rotation of the cross-arm $\theta_{c}$, which is given by:

$$
\theta_{c}=\left[\arcsin \left(Q_{2}-Q_{3}\right)-\arcsin \left(Q_{2}-Q_{1}\right)\right] / 2,
$$

and assuming that any bending in the cross-arm is negligible, gives the squares of the lengths of the stays thus:

$$
\begin{aligned}
& L_{s 1}^{2}=\left[\left(a \cos \theta_{c}+\left|Q_{2}\right| l\right)^{2}+\left(L_{v 1}+a \sin \theta_{c}\right)\right], \\
& L_{s 2}^{2}=\left[\left(a \cos \theta_{c}+\left|Q_{2}\right| l\right)^{2}+\left(L_{v 2}-a \sin \theta_{c}\right)\right], \\
& L_{s 3}^{2}=\left[\left(a \cos \theta_{c}-\left|Q_{2}\right| l\right)^{2}+\left(L_{v 2}+a \sin \theta_{c}\right)\right], \\
& L_{s 4}^{2}=\left[\left(a \cos \theta_{c}-\left|Q_{2}\right| l\right)^{2}+\left(L_{v 1}-a \sin \theta_{c}\right)\right] .
\end{aligned}
$$

Cable slackening is defined to occur when:

$$
L_{s i}^{2} \leqslant\left(a^{2}+4 l^{2}\right)\left[1+\frac{T}{E_{s} A_{s}}\right]^{-2},
$$

where $E_{s}, A_{s}$ and $a$ are defined earlier and $T$ is the prestressing tensile force in the stays prior to loading. The expression in equation (17) is given by using Pythagoras's Theorem for the cable stays once the link model exhibits buckling displacements.

\section{Results and discussion}

The potential energy is expressed in the exact form and the respective equilibrium equations are given by the following relation:

$$
\frac{\partial V}{\partial Q_{i}}=0
$$

where $i=1,2,3$, which leads to three nonlinear algebraic equations that are solved numerically in Auto.

\subsection{Perfect post-buckling response}

For the unrestrained uniform strut case, where $R=1$ and $K=0$, there are three distinct critical loads $p_{i}^{\mathrm{C}}$ and the post-buckling response is weakly stable for the lowest (symmetric) mode, as would be expected from knowledge of the elastica. When the translational spring is introduced into the model, the critical loads for Modes 1 and 3 increase with $K$, but the critical load for Mode 2 remains constant owing to the pin at $C$ remaining static. Figure 7 shows a series of solutions for the equilibrium equations where Mode 1 is critical. The post-buckling path of the symmetric 
mode becomes less stable and in fact the response becomes subcritical when $K \approx 0.3$. However, even when the post-buckling becomes unstable, there is no nonlinear interaction with higher modes, which implies that it is only sensitive to imperfections that are affine to the eigenmode of Mode 1. Note that, similar to the linear eigenvalue solution, the path for Mode 2 remains invariant and stable.

When $K=2$ the critical loads for Modes 1 and 2 coincide, and for larger values of $K$ Mode 2 becomes critical. However, as seen in Figures 8 where $K=2.01$, the post-buckling path of the Mode 2 is destabilized at a secondary bifurcation point $\mathrm{S}_{2}$ where the response transfers to a path where the modes interact, the antisymmetry is broken and eventually passes back to the subcritical symmetric mode through a further bifurcation point $S_{3}$. This entire process has been termed mode jumping in the literature (Hunt \& Everall, 1999). Examining Figure 8, an elliptical mode interaction loop is observed between the modal amplitudes of $Q_{1}$ and $Q_{2}$ on which the secondary bifurcations are found. This interaction loop defines combinations of the relevant amplitudes where the symmetry of the linear eigenmodes is broken at subsequent secondary instabilities and a pathway is found where one eigenmode can in fact be transformed into another.

Increasing $K$ further causes the interaction loop to increase in size, hence the secondary instabilities become remote from the primary ones and interaction is seen less readily. Once the amplitudes of $Q_{i}$ reach nearer unity (its physical limit), the loop begins to distort significantly as seen in Figure 9, which shows the solutions for two further examples of $K$ being 2.1 and 2.6 respectively. Of course if the loop breaks, as seen in Figure 9(b), once the mode jumps to the interactive asymmetric mode, the structure would remain in that configuration. This finding resonates with a previous finite element study by Saito \& Wadee (2009b), where post-buckling in Mode 2 was found to "prefer" to deform in the asymmetric mode, rather than the linear eigenmode. Recently conducted physical experiments also corroborate this finding (Osofero et al., 2012) where columns designed to trigger Mode 2 showed interactive post-buckling behaviour, whereas those designed to trigger Mode 1 showed deformation in the linear eigenmode.

\subsection{Effects of stay slackening}

To demonstrate the effects of stay slackening, certain properties to be assumed. The length of the main column element $L$ is assumed to be $4 \mathrm{~m}$, hence $l=1 \mathrm{~m}$. The cross-arm length $a$ is assumed to be equal to $l$, which would usually cause Mode 2 to be the lowest eigenmode. The level of the prestressing force $T=10 \mathrm{kN}$ with the cable stay having $A_{s}=50 \mathrm{~mm}^{2}$ and $E_{s}=210 \mathrm{kN} / \mathrm{mm}^{2}$. It is also assumed that the contribution to the stiffness of each stay is equal; hence each stay going slack reduces the total stiffness of the combined initial stay stiffnesses, which contribute to $K$, by $25 \%$. Pure eigenmodes cause slacking in stay pairs (an immediate $50 \%$ reduction of $K$ ), whereas interactive buckling causes slackening in individual stays (an immediate $25 \%$ reduction of $K$ ).

Figure 10 shows the post-buckling paths for Modes 1-3 for three cases where $K<2$. Obviously, the case where $K=0$ is unchanged, but the other cases show an immediate loss in the load-carrying capacity after the initial instability. However, as before, no mode interaction is shown for these cases. Figure 11 shows the post-buckling paths for the case where $K=2.01$, which exhibit a far more severe response compared to Figure 8, with the secondary instabilities 
being triggered at much smaller values of $Q_{1}$. The response, as before first triggers Mode 2 at point $S_{1}$, then an interactive mode at point $S_{2}$ and finally triggering Mode 1 at point $S_{3}$. Figure 12 shows results for $K=4.10$, with the post-buckling paths showing a similar pattern to the case for $K=2.01$, although the interaction loop is severely distorted. Finally, Figure 13 shows results for $K=4.82$; the post-buckling paths initially display a similar pattern to the cases for $K=2.01$ and $K=4.10$. However, this time the interaction loop is broken and the response remains in the interactive mode. This finding is similar to that shown in Figure 9(b) but at much smaller combinations of the amplitudes of $Q_{1}$ and $Q_{2}$. This implies that the effects of cable slackening, even though it has been modelled with certain simplifying assumptions, is shown to be significant and forces the response to remain in the interactive mode. This has been observed in the nonlinear modelling work on prestressed stayed tubular columns using the finite element method (Saito \& Wadee, 2009b) and from recently conducted experiments (Osofero et al., 2012), which demonstrates that the current model provides an excellent tool for understanding the complex mechanical phenomena predicted by finite element analysis and physical experiments.

\section{Concluding remarks}

A simplified theoretical model, comprising rigid links and springs formulated using potential energy principles, has been presented to simulate the practically observed interactive buckling phenomena in prestressed stayed columns. Moreover, the destabilizing effect of the slackening of cable stays has been included from a geometric criterion. It is found that, in line with observations in practice, the link model only exhibits interactive buckling if the initial instability triggers the antisymmetric eigenmode. Moreover, it is found that if cable stay slackening is included then the secondary instability leading to mode interaction occurs at significantly smaller values of the generalized coordinates than if slackening is neglected.

This type of model has the potential for being extended to multi-bay prestressed stayed columns, which have more than one cross-arm, an example of which is shown in Figure 1(b). In those cases, as far as the authors are aware, no relevant nonlinear studies exist and so it is currently unclear which modes may interact. Given that the current study has been shown to replicate the behaviour for the single cross-arm case, it is suggested that a similar result may be forthcoming for more complex arrangements of this efficient load carrying structural member. This would then be able to support the formulation of design guidance (Wadee et al., 2013), as has been developed recently for the single cross-arm case.

\section{Acknowledgement}

This work was funded by the UK Engineering and Physical Sciences Research Council (EPSRC) through the research project reference: EP/F022182/1. 


\section{A Diagonalization of $V$}

Assuming that the buckling mode shape to be a linear superposition of the eigenmodes shown in Figure 4 and denoting the actual shape as $S$ and the normalized eigenmodes as $m_{1}, m_{2}$ and $m_{3}$ for modes 1-3 respectively, where

$$
S=\sum_{n=1}^{3} m_{n} u_{n}
$$

where $u_{n}$ are variables that indicate the proportion of the $n$th eigenmode, thus:

$$
\begin{aligned}
& Q_{1}=u_{1}+u_{2}+u_{3}, \\
& Q_{2}=\lambda_{1} u_{1}-\lambda_{3} u_{3}, \\
& Q_{3}=u_{1}-u_{2}+u_{3} .
\end{aligned}
$$

Substituting these relationships into the nondimensional potential energy function $\tilde{V}$ given in Equation (6) yields a lengthy expression which is not presented for brevity. Once this is expressed to leading order, the expression being purely in terms of quadratic terms in $u_{i} u_{j}$, the diagonalization may commence by finding the relationships for $\lambda_{1}$ and $\lambda_{3}$ that eliminate the quadratic cross terms where $i \neq j$; these are:

$$
\begin{aligned}
& \lambda_{1}=\frac{2(R-1)+K-\sqrt{4\left(R^{2}+1\right)-4 K+K^{2}}}{K-2}, \\
& \lambda_{3}=\frac{2(R-1)+K+\sqrt{4\left(R^{2}+1\right)-4 K+K^{2}}}{K-2} .
\end{aligned}
$$

Clearly, there is a singularity when $K=2$. This change of variables from $Q_{i}$ to $u_{i}$, yields a diagonalized energy function and the linear eigenvalue problem yields the following condition for critical equilibrium, thus:

$$
\left(\frac{\partial^{2} \tilde{V}}{\partial u_{1}^{2}}\right)\left(\frac{\partial^{2} \tilde{V}}{\partial u_{2}^{2}}\right)\left(\frac{\partial^{2} \tilde{V}}{\partial u_{3}^{2}}\right)=0,
$$

the solutions of which are given in equations (7)-(9) for the case where $R=1$.

\section{References}

Andrade, S. A. L. de, Vellasco, P. C. G. da S., \& Silva, J. G. S. da. 2003. Concepcão e projecto estrutural do palco principal do Rock in Rio III. Construçao Magazine, 6, 4-11. (in Portuguese).

Belenya, E. 1977. Prestressed load-bearing metal structures. Moscow: Mir Publishers.

Chan, S. L., Shu, G., \& Lü, Z. 2002. Stability analysis and parametric study of pre-stressed stayed columns. Eng. Struct., 24, 115-124. 
Coates, R. C., Coutie, M. G., \& Kong, F. K. 1987. Structural analysis. London: Taylor and Francis.

De Araujo, R. R., De Andrade, S. A. L., Vellasco, P. C. G. da. S., \& Da Silva, J. G. S.and De Lima, L. R. O. 2008. Experimental and numerical assessment of stayed steel columns. J. Constr. Steel Res., 64(9), 1020-1029.

Doedel, E. J., \& Oldeman, B. E. 2009. AUTO-07p: Continuation and bifurcation software for ordinary differential equations. Tech. rept. Department of Computer Science, Concordia University, Montreal, Canada. Available from http://indy.cs.concordia.ca/auto/.

Hafez, H. H., Temple, M. C., \& Ellis, J. S. 1979. Pretensioning of single-crossarm stayed columns. J. Struct. Div.-ASCE, 105(ST 2), 359-375.

Howson, W. P., \& Williams, F. W. 1980. A parametric study of the initial buckling of stayed columns. Proc. Instn Civ. Engrs., 69, 261-279.

Hunt, G. W., \& Everall, P. R. 1999. Arnol'd tongues and mode jumping in the supercritical post-buckling of an archetypal elastic structure. Proc. R. Soc. A, 455(1981), 125-140.

Osofero, A. I., Wadee, M. A., \& Gardner, L. 2012. Experimental study of critical and postbuckling behaviour of prestressed stayed columns. J. Constr. Steel Res., 79, 226-241.

Osofero, A. I., Wadee, M. A., \& Gardner, L. 2013. Numerical studies on the buckling resistance of prestressed stayed columns. Adv. Struct. Eng., 16(3), 487-498.

Saito, D., \& Wadee, M. A. 2008. Post-buckling behaviour of prestressed steel stayed columns. Eng. Struct., 30(5), 1224-1239.

Saito, D., \& Wadee, M. A. 2009a. Buckling behaviour of prestressed steel stayed columns with imperfections and stress limitation. Eng. Struct., 31(1), 1-15.

Saito, D., \& Wadee, M. A. 2009b. Numerical studies of interactive buckling in prestressed steel stayed columns. Eng. Struct., 31(2), 432-443.

Saito, D., \& Wadee, M. A. 2010. Optimal prestressing and configuration of prestressed stayed columns. Proc. Inst. Civil Eng. - Struct. Build., 163(SB5), 343-355.

Seward, A. 2011. In detail: Frick Chemistry Laboratory. http: / / archpaper . com/news / articles.asp?id=5303.

Smith, R. J., McCaffrey, G. T., \& Ellis, J. S. 1975. Buckling of a single-crossarm stayed column. J. Struct. Div. ASCE., 101(ST1), 249-268.

Temple, M. C. 1977. Buckling of stayed columns. J. Struct. Div. ASCE., 103(ST4), 839-851. 
Wadee, M. A., Gardner, L., \& Osofero, A. I. 2013. Design of prestressed stayed columns. J. Constr. Steel Res., 80, 278-287.

Wong, K. C., \& Temple, M. C. 1982. Stayed column with initial imperfection. J. Struct. Div. ASCE., 108(ST7), 1623-1640. 


\section{List of Figures}

1 (a) Prestressed stayed column arrangement. (b) Prestressed stayed tubular column in the Chiswick Park development in West London, UK. . . . . . . . . . . . 14

2 Critical buckling loads with (a) the stay diameter $\phi_{s}$ varying and (b) cross-arm length $a$ varying. These graphs are taken from the study by Saito \& Wadee (2009b) where the length of a specific stayed column was fixed. The cases F1-F6 and a1-a10 represent individual configurations for stay diameters and cross-arm lengths respectively in that article. . . . . . . . . . . . . . . . 15

3 (a) Model with rigid links and springs; (b) continuous and discrete eigenmodes. . 16

4 Linear eigenmodes. The quantities of $\lambda_{1}$ and $\lambda_{3}$ depend on the magnitude of the translational spring stiffness $k$. When $k=0, \lambda_{1}=-\lambda_{3}=\sqrt{2}$. As $k \rightarrow \infty$,

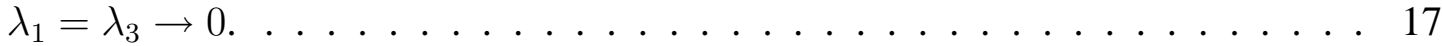

5 Distribution of critical loads with the non-dimensional stiffness of the translational spring $K$; note that $R=1$. When $K>2$, Mode 2 has the lowest critical load. . . . . . . . . . . . . . . . . . . . . . 18

6 (a) Number definition of cable stays. (b) Asymmetric buckling mode used to define cable stay lengths under loading. . . . . . . . . . . . . . . . .

7 Post-buckling paths for $K=0,0.9$ and 1.8 where Mode 1 is critical. Note that the critical load associated with the first encountered eigenmode increases but that its unstable nature becomes more severe. . . . . . . . . . . . . . . . .

8 Post-buckling response $p$ versus $Q_{1}$ (top) and $Q_{2}$ versus $Q_{1}$ (bottom) for the discrete strut model when $K=2.01$. Point $\mathrm{S}_{1}$ is the critical bifurcation point, whereas points $\mathrm{S}_{2}$ and $\mathrm{S}_{3}$ are secondary bifurcation points on the post-buckling paths of the antisymmetric and symmetric modes respectively. The path connecting these two bifurcations contain equilibrium configurations where the geometric symmetry of the strut model is broken. The elliptical mode interaction loop is shown clearly. . . . . . . . . . . . . . . . . . . .

9 Mode interaction loops for higher $K$ values shown in $Q_{1} Q_{2}$ space. Top: $K=$ 2.1 ; bottom: $K=2.6$. Note the significant distortion due to $Q_{1}$ and $Q_{2}$ reaching their physical limits for the higher $K$ value. . . . . . . . . . . . .

10 Post-buckling paths for $K=0,0.9$ and 1.8 where Mode 1 is critical for model including stay slackening. Note the sudden drop in the load-carrying capacity beyond the lowest bifurcation point and the lack of subsequent mode interaction.

11 Plots of normalized load $p$ versus $Q_{1}$ and $Q_{2}$ versus $Q_{1}$ for $K=2.01$. Note that the stay slackening causes the secondary instabilities to be relatively closer to the primary one and the interaction loop to be slightly distorted from the elliptical

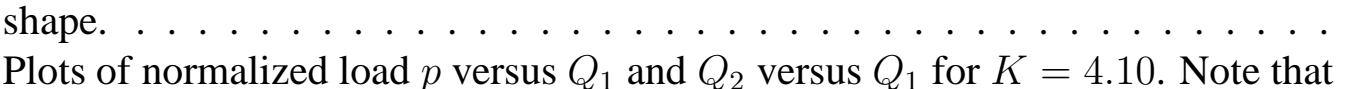
the stay slackening causes the interaction loop to be severely distorted. . . . . . . 25 
13 Plots of normalized load $p$ versus $Q_{1}$ and $Q_{2}$ versus $Q_{1}$ for $K=4.82$. Note that the level of the stiffness and the stay slackening causes a rapid reduction in the load-carrying capacity, the interaction loop to be broken and the structure to remain in the interactive mode. . . . . . . . . . . . 26 

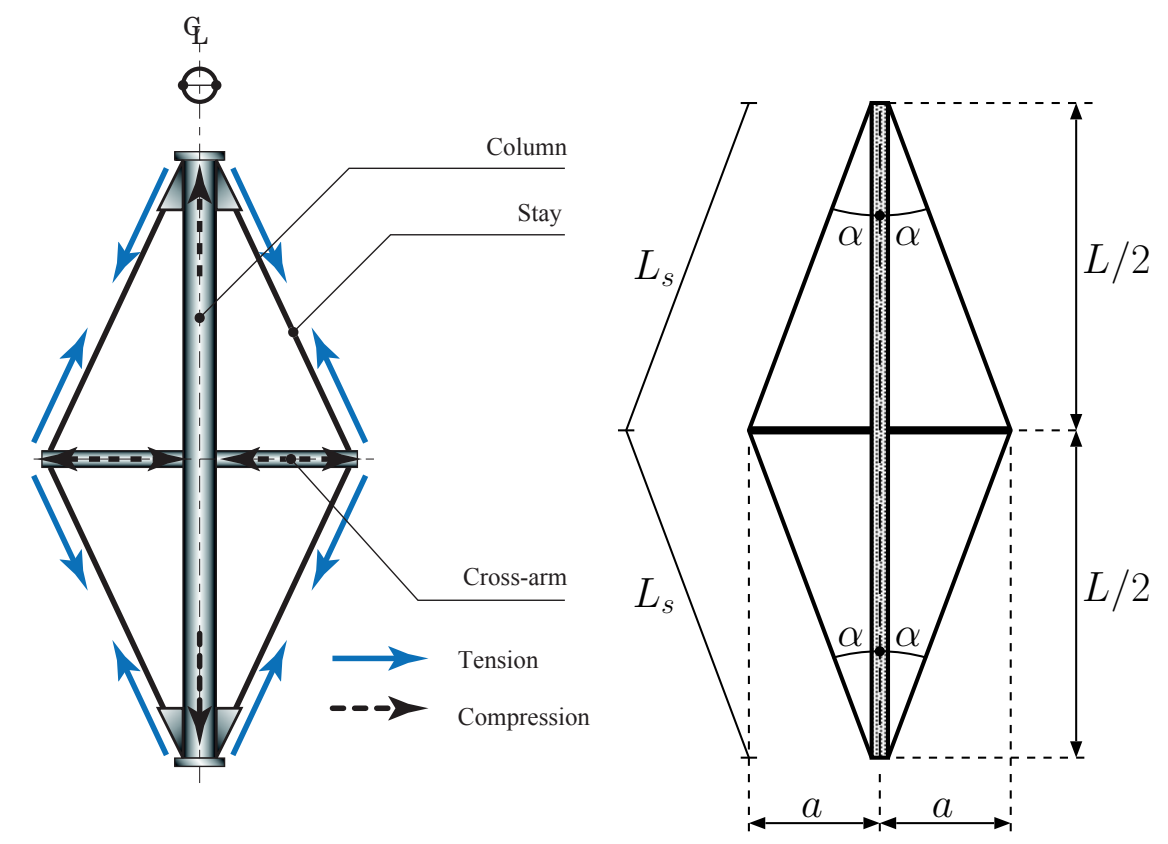

(a)

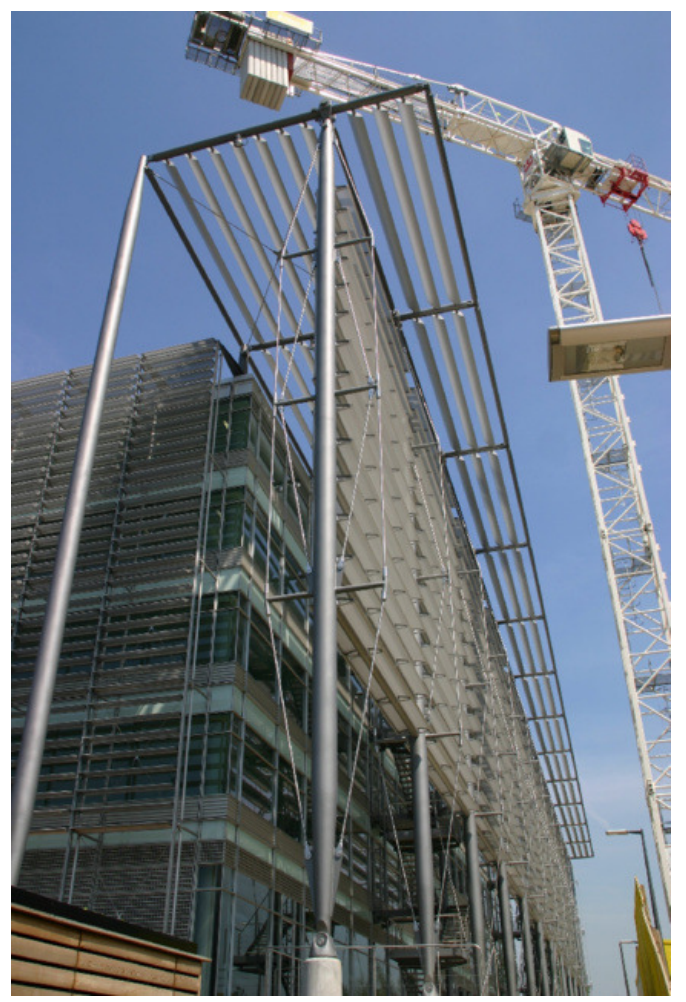

(b)

Figure 1: (a) Prestressed stayed column arrangement. (b) Prestressed stayed tubular column in the Chiswick Park development in West London, UK. 


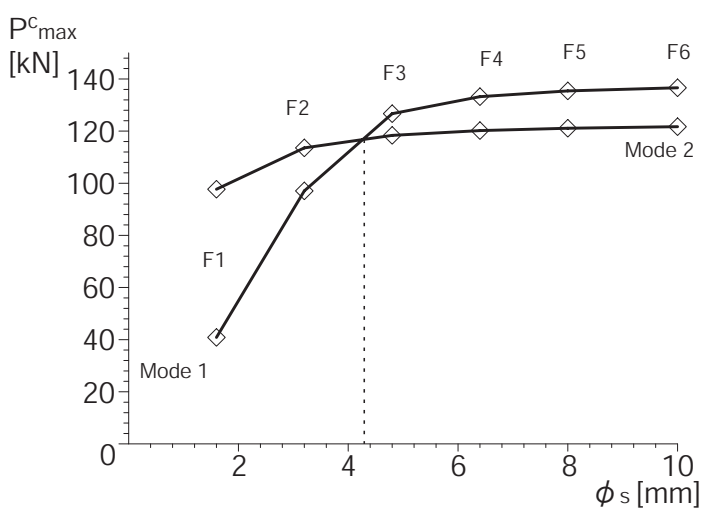

(a)

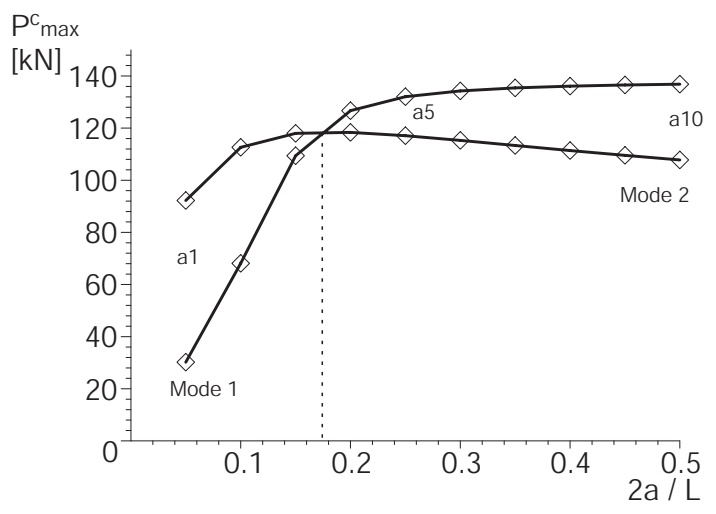

(b)

Figure 2: Critical buckling loads with (a) the stay diameter $\phi_{s}$ varying and (b) cross-arm length $a$ varying. These graphs are taken from the study by Saito \& Wadee (2009b) where the length of a specific stayed column was fixed. The cases F1-F6 and a1-a10 represent individual configurations for stay diameters and cross-arm lengths respectively in that article. 


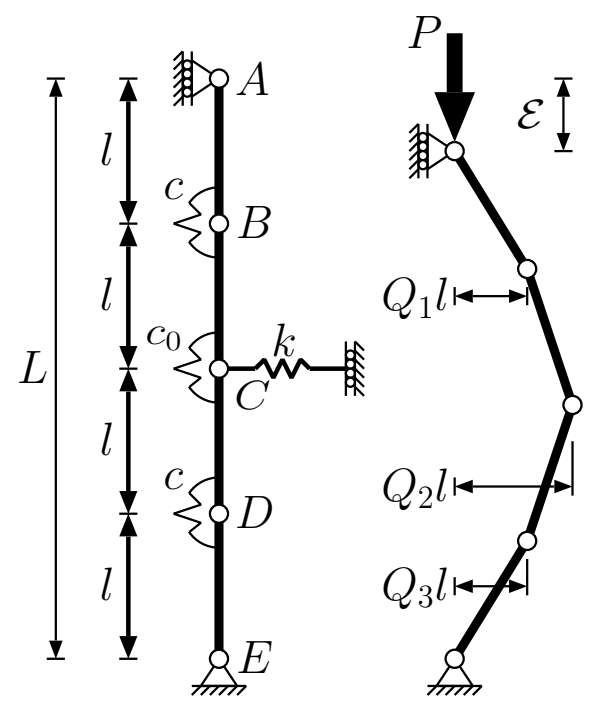

(a) Discrete model: 3-DOF

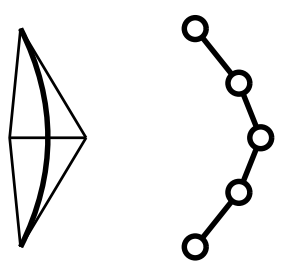

Symmetric mode (Mode 1)

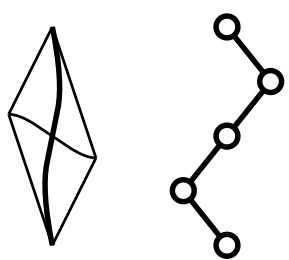

Antisymmetric mode (Mode 2)

(b) Eigenmodes

Figure 3: (a) Model with rigid links and springs; (b) continuous and discrete eigenmodes. 


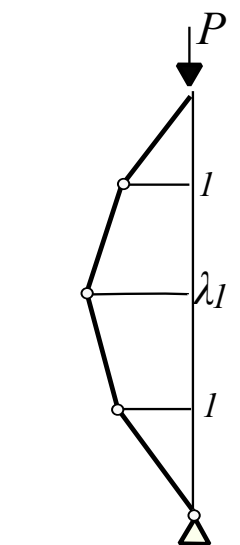

(a) Mode 1

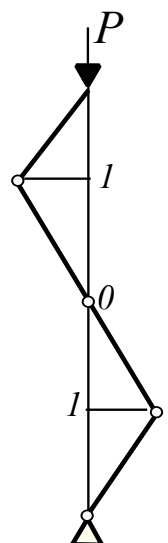

(b) Mode 2

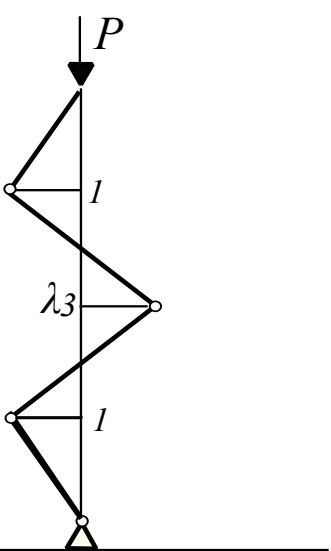

(c) Mode 3

Figure 4: Linear eigenmodes. The quantities of $\lambda_{1}$ and $\lambda_{3}$ depend on the magnitude of the translational spring stiffness $k$. When $k=0, \lambda_{1}=-\lambda_{3}=\sqrt{2}$. As $k \rightarrow \infty, \lambda_{1}=\lambda_{3} \rightarrow 0$. 


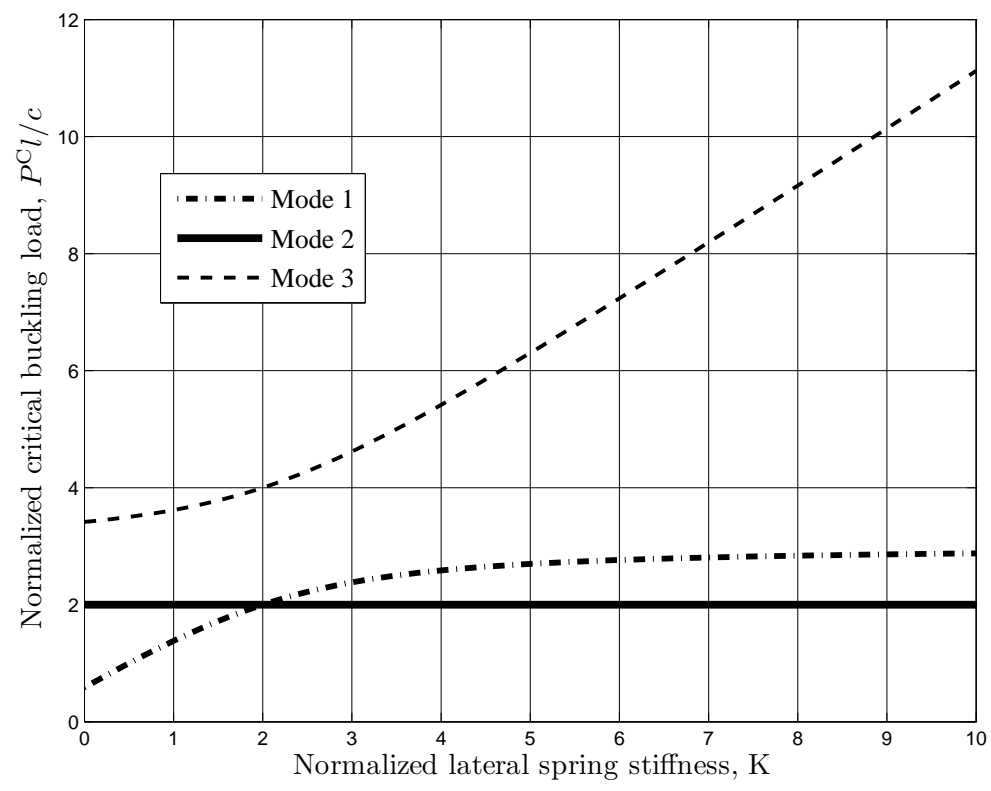

Figure 5: Distribution of critical loads with the non-dimensional stiffness of the translational spring $K$; note that $R=1$. When $K>2$, Mode 2 has the lowest critical load. 


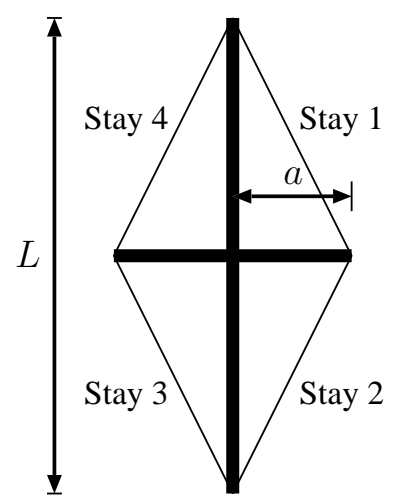

(a)

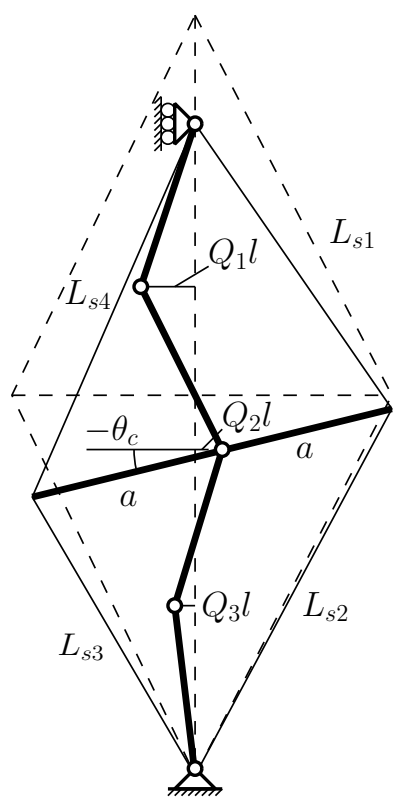

(b)

Figure 6: (a) Number definition of cable stays. (b) Asymmetric buckling mode used to define cable stay lengths under loading. 


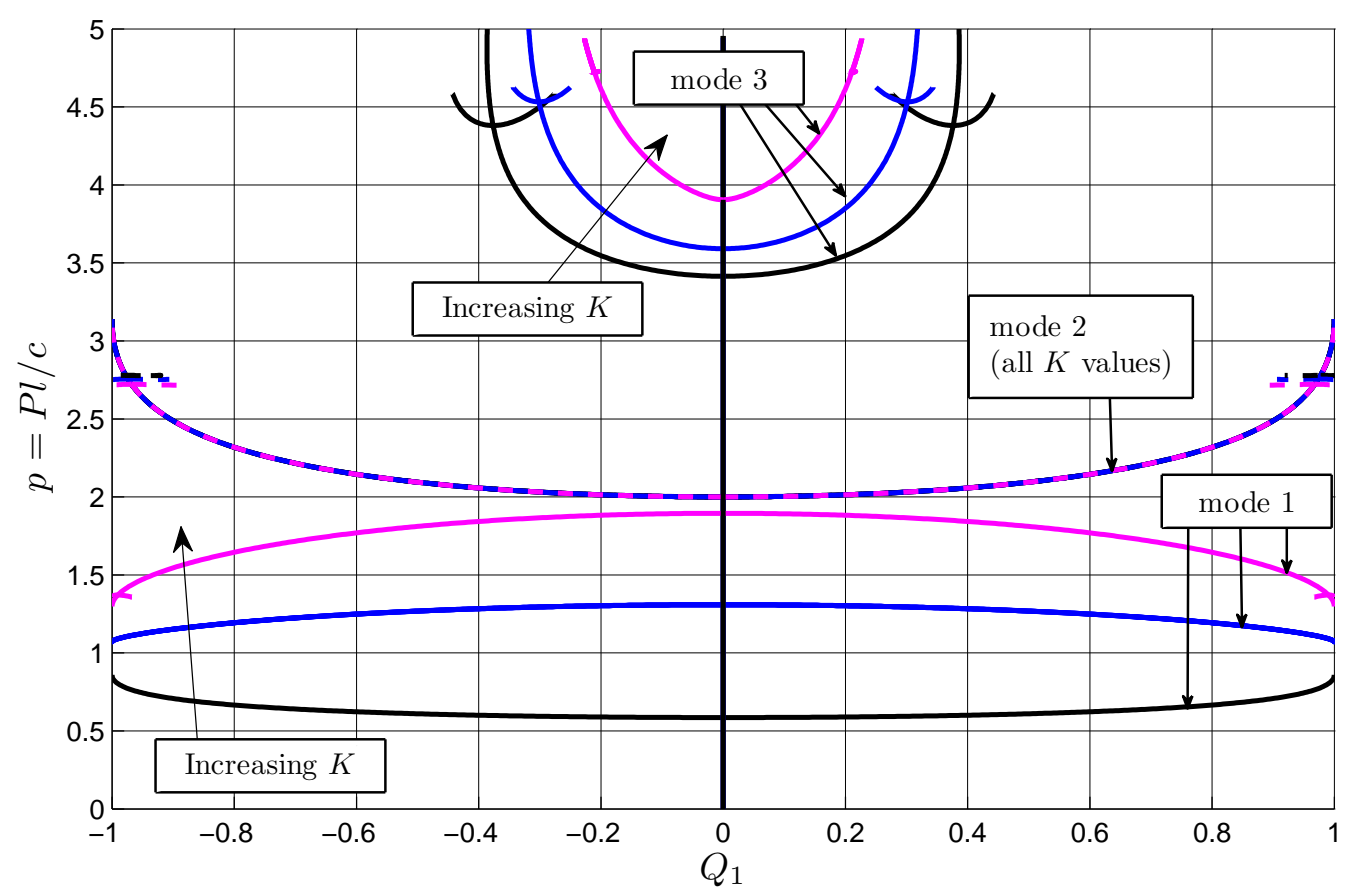

Figure 7: Post-buckling paths for $K=0,0.9$ and 1.8 where Mode 1 is critical. Note that the critical load associated with the first encountered eigenmode increases but that its unstable nature becomes more severe. 

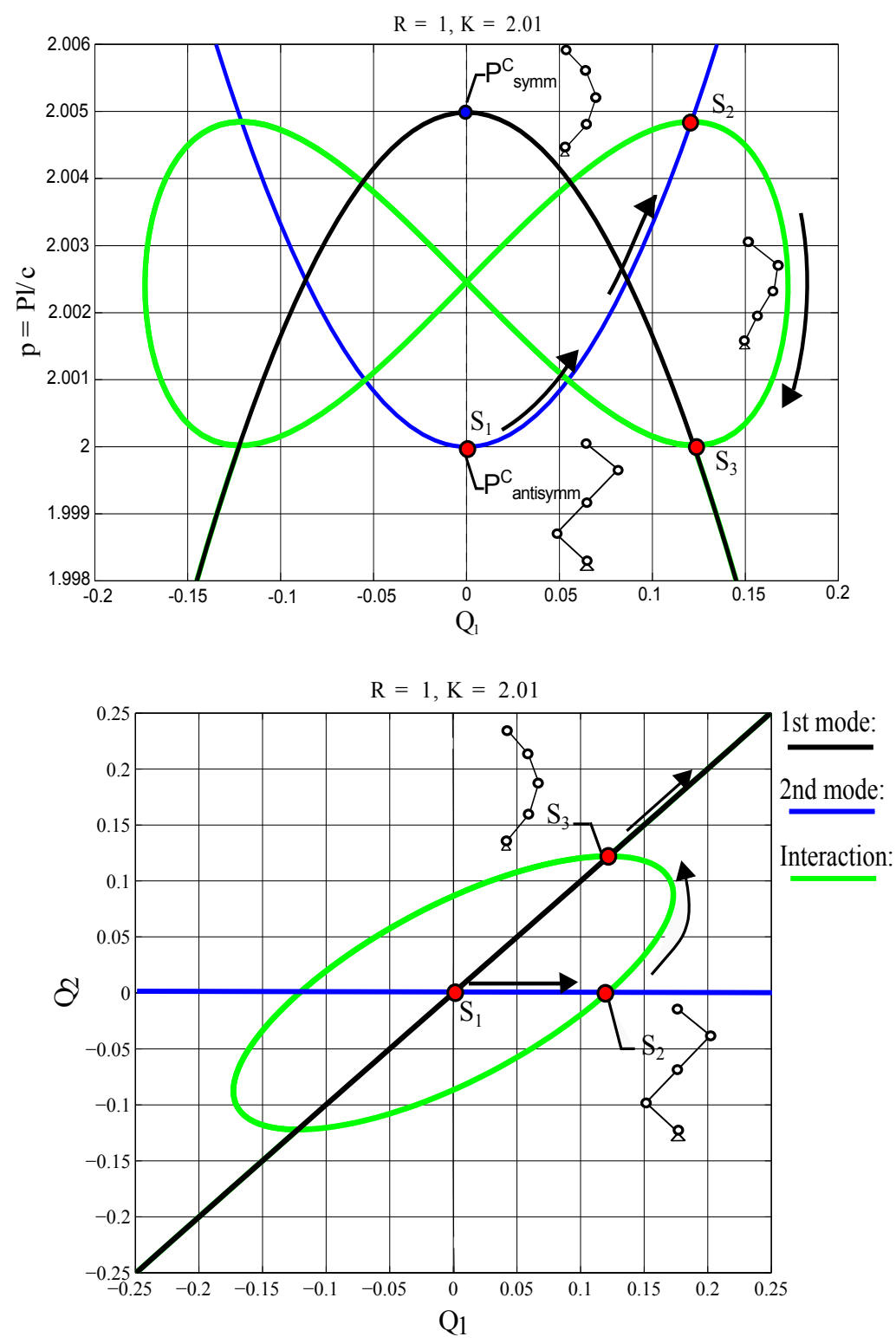

Figure 8: Post-buckling response $p$ versus $Q_{1}$ (top) and $Q_{2}$ versus $Q_{1}$ (bottom) for the discrete strut model when $K=2.01$. Point $S_{1}$ is the critical bifurcation point, whereas points $S_{2}$ and $S_{3}$ are secondary bifurcation points on the post-buckling paths of the antisymmetric and symmetric modes respectively. The path connecting these two bifurcations contain equilibrium configurations where the geometric symmetry of the strut model is broken. The elliptical mode interaction loop is shown clearly. 

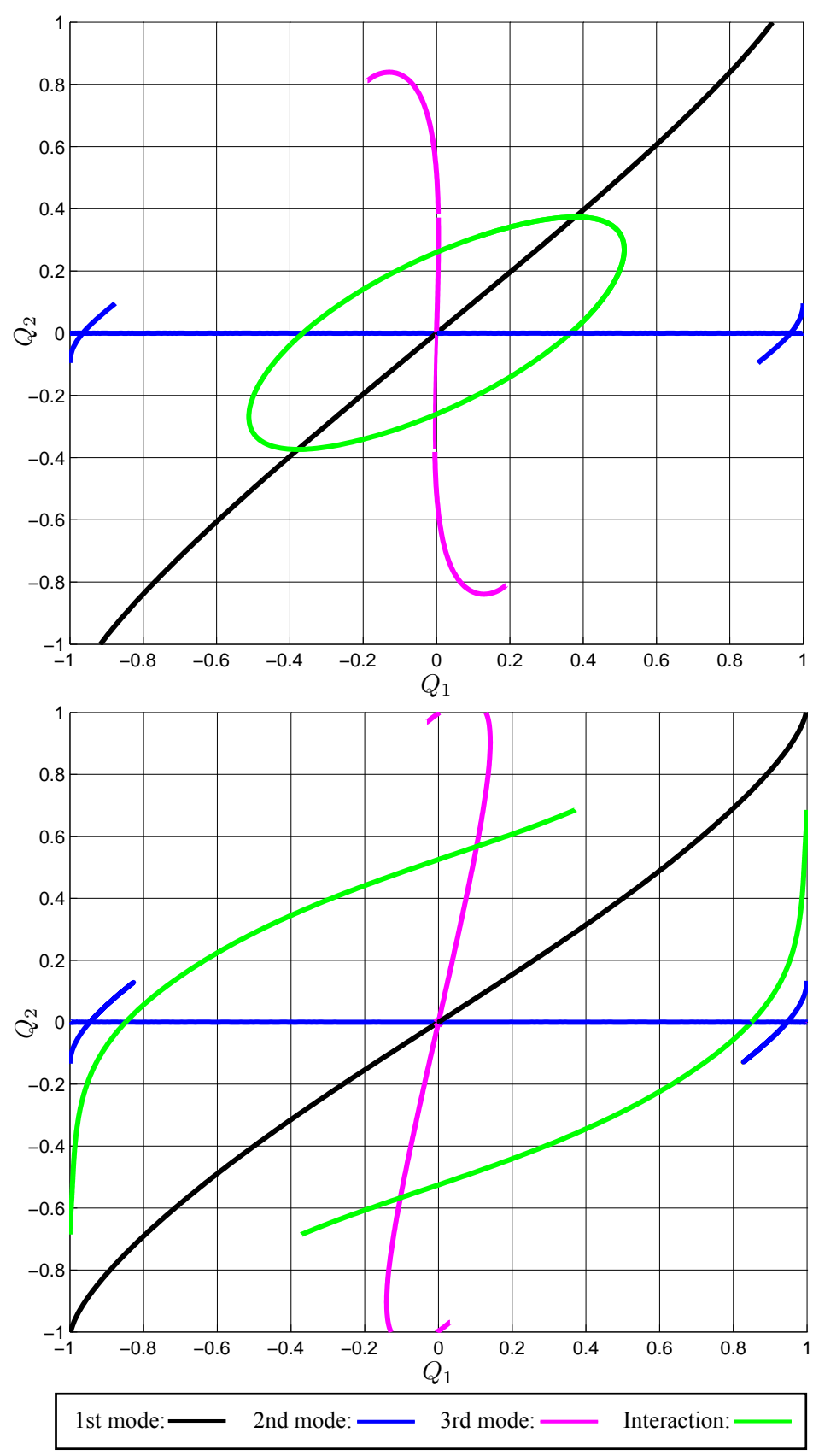

Figure 9: Mode interaction loops for higher $K$ values shown in $Q_{1} Q_{2}$ space. Top: $K=2.1$; bottom: $K=2.6$. Note the significant distortion due to $Q_{1}$ and $Q_{2}$ reaching their physical limits for the higher $K$ value. 


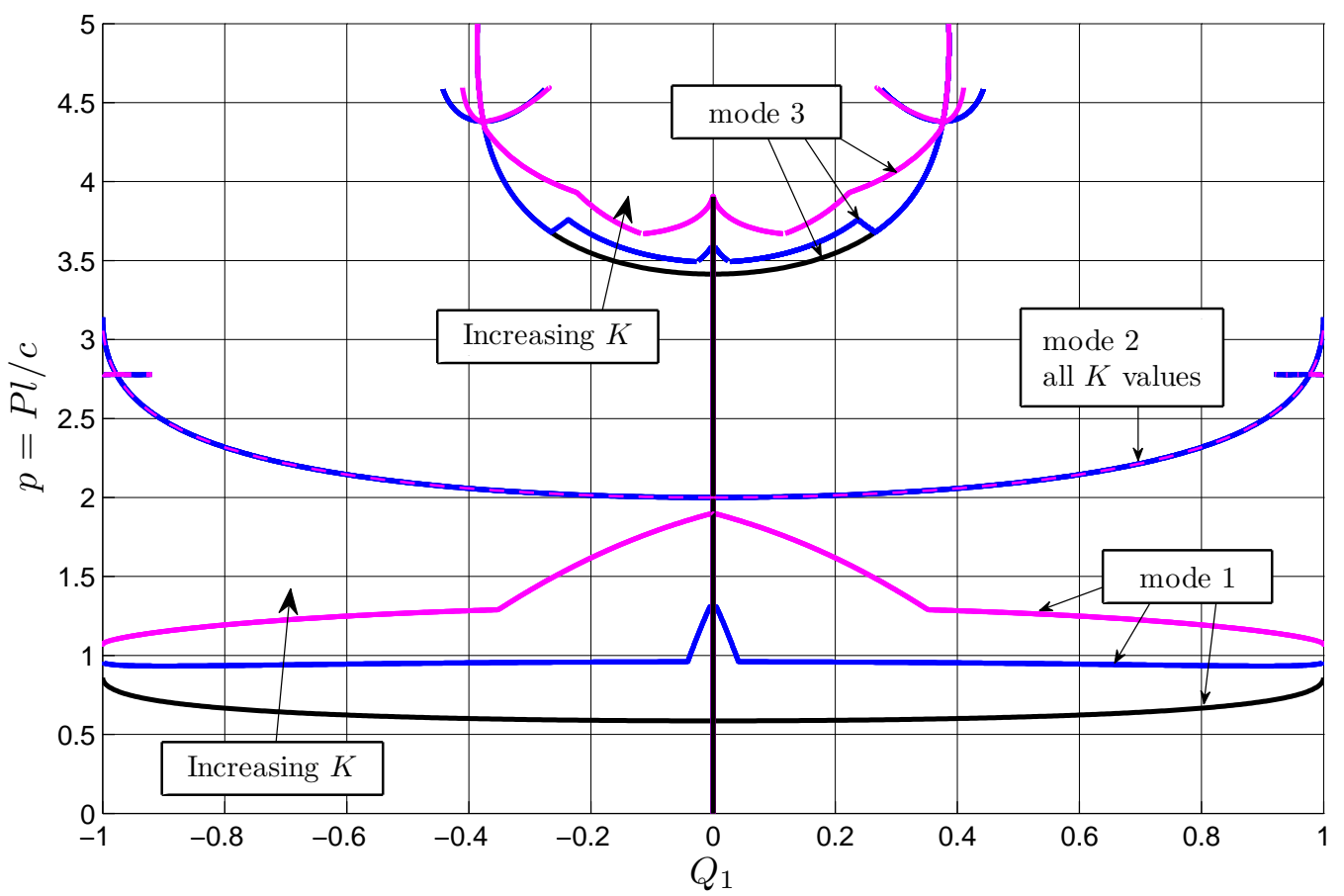

Figure 10: Post-buckling paths for $K=0,0.9$ and 1.8 where Mode 1 is critical for model including stay slackening. Note the sudden drop in the load-carrying capacity beyond the lowest bifurcation point and the lack of subsequent mode interaction. 

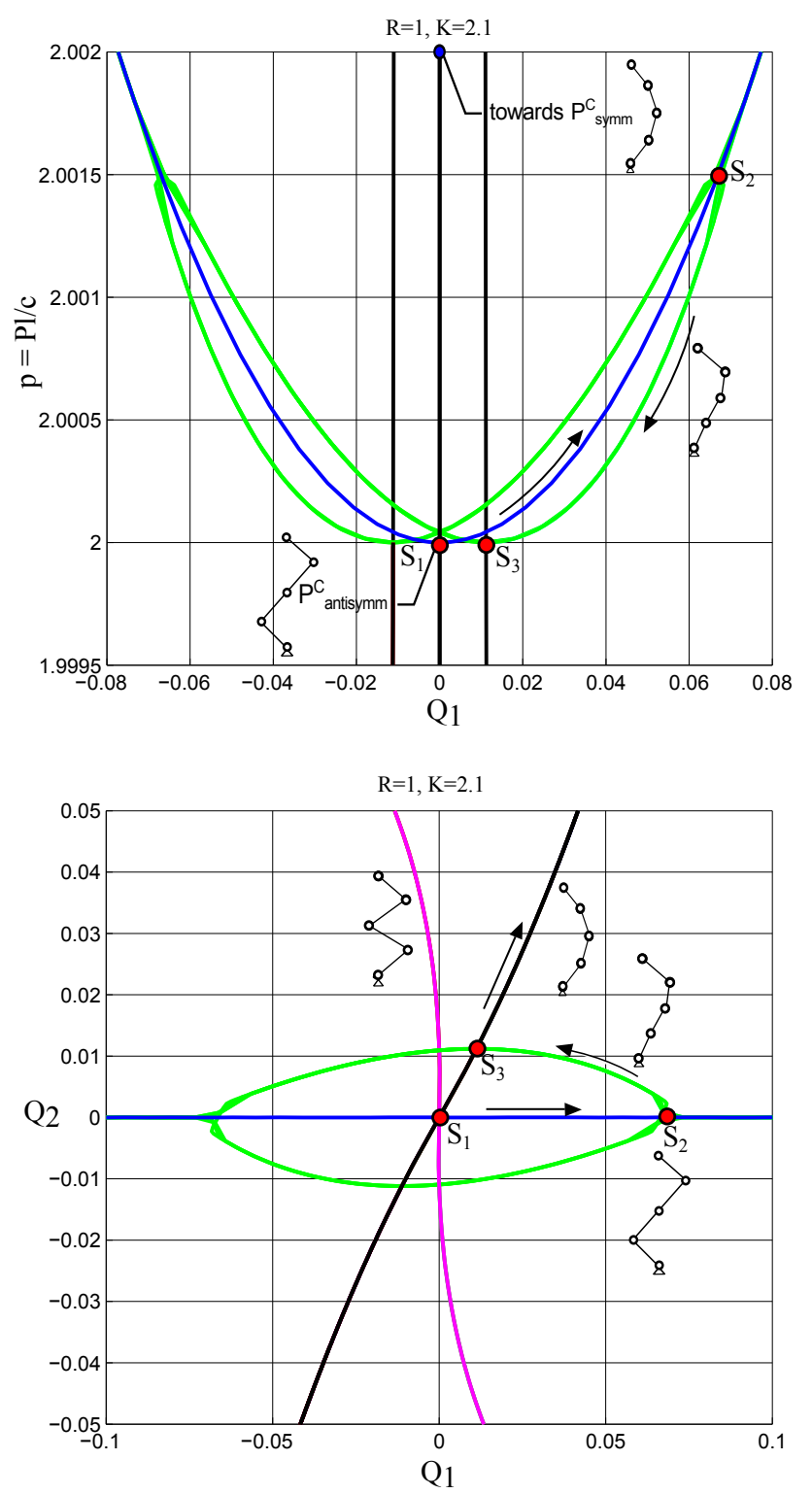

1st mode: $\longrightarrow$ 2nd mode: $\longrightarrow$ 3rd mode: $\longleftarrow$ Interaction: -

Figure 11: Plots of normalized load $p$ versus $Q_{1}$ and $Q_{2}$ versus $Q_{1}$ for $K=2.01$. Note that the stay slackening causes the secondary instabilities to be relatively closer to the primary one and the interaction loop to be slightly distorted from the elliptical shape. 

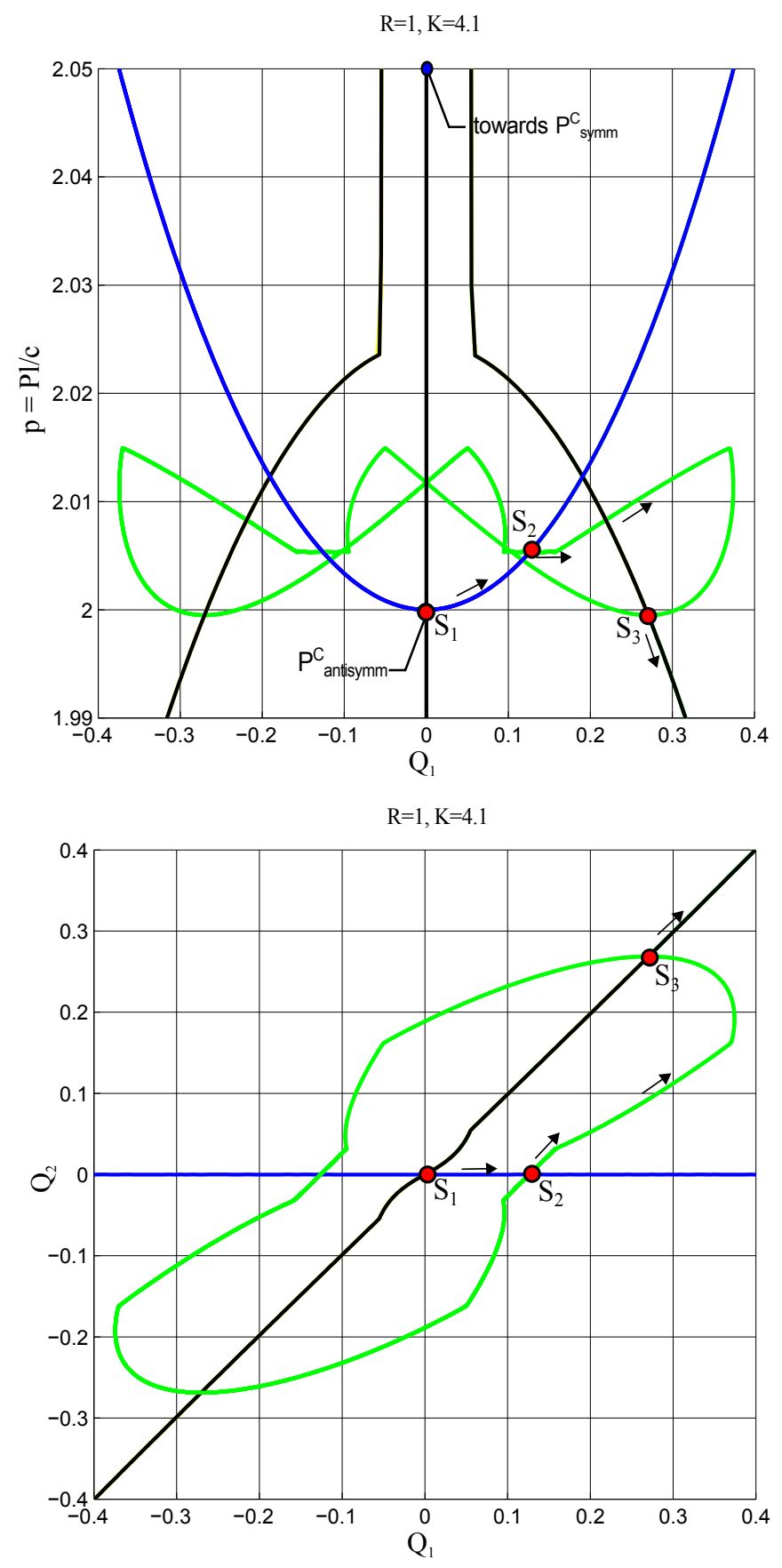

1st mode: $\longrightarrow \quad$ 2nd mode: $\longrightarrow \quad$ Interaction: $=$

Figure 12: Plots of normalized load $p$ versus $Q_{1}$ and $Q_{2}$ versus $Q_{1}$ for $K=4.10$. Note that the stay slackening causes the interaction loop to be severely distorted. 

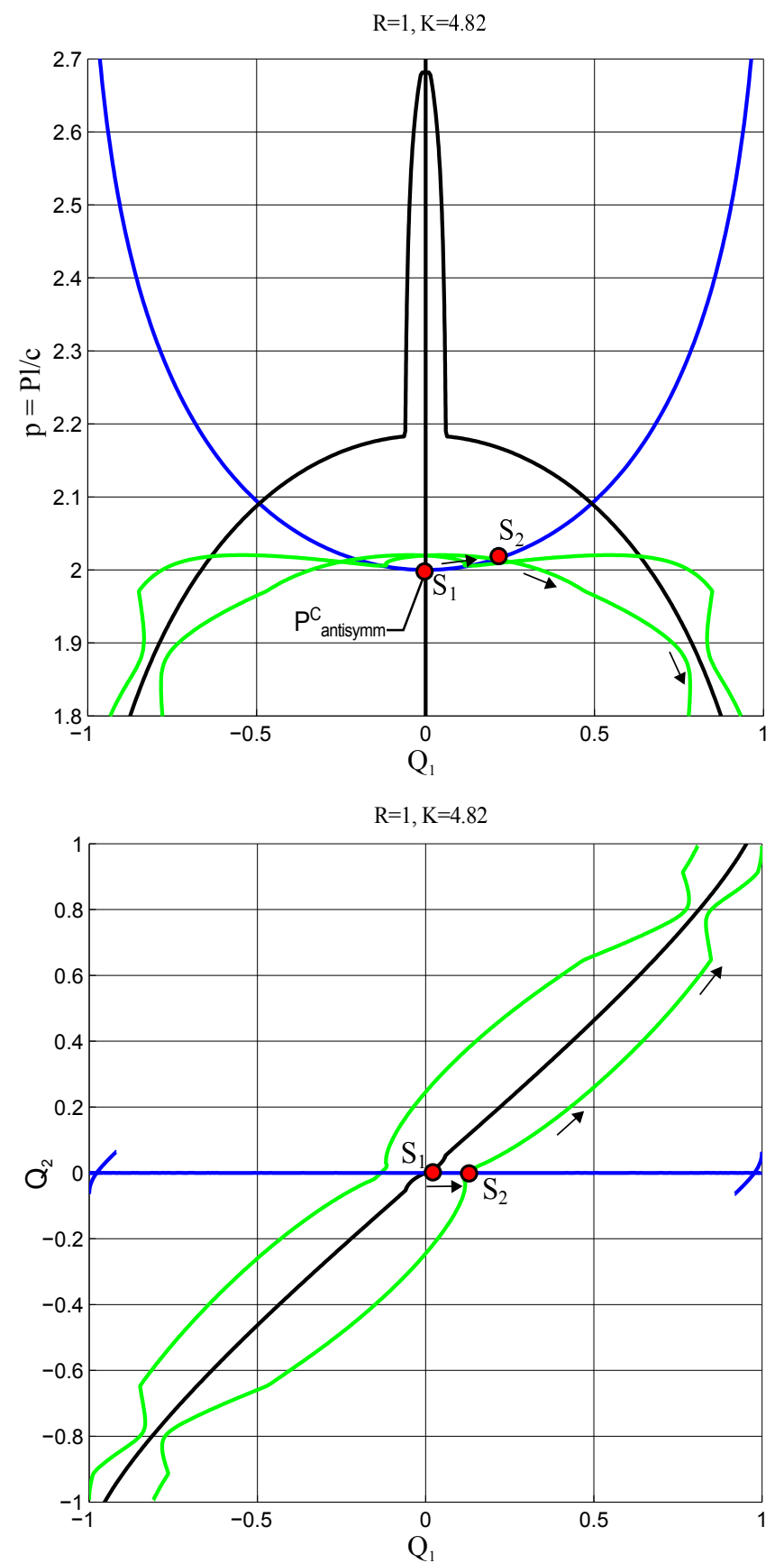

1 st mode

2nd mode

Interaction

Figure 13: Plots of normalized load $p$ versus $Q_{1}$ and $Q_{2}$ versus $Q_{1}$ for $K=4.82$. Note that the level of the stiffness and the stay slackening causes a rapid reduction in the load-carrying capacity, the interaction loop to be broken and the structure to remain in the interactive mode. 